\title{
Midlife adiposity predicts cognitive decline in the prospective Multicenter AIDS Cohort Study
}

Leah H. Rubin, PhD, MPH, Deborah Gustafson, PhD, Kellie L. Hawkins, MD, Long Zhang, PhD, Lisa P. Jacobson, PhD, James T. Becker, PhD, Cynthia A. Munro, PhD, Jordan E. Lake, MD, Eileen Martin, PhD, Andrew Levine, PhD, Todd T. Brown, MD, Ned Sacktor, MD, and Kristine M. Erlandson, MD

Neurolog ${ }^{\circledR}$ 2019;93:e261-e271. doi:10.1212/WNL.0000000000007779

\section{Abstract}

\section{Objective}

Obesity is a common, modifiable cardiovascular and cerebrovascular risk factor. Among people with HIV, obesity may contribute to multisystem dysregulation including cognitive impairment. We examined body mass index (BMI) and central obesity (waist circumference [WC]) in association with domain-specific cognitive function and 10-year cognitive decline in men with HIV infection (MWH) vs HIV-uninfected (HIV-) men.

\section{Methods}

A total of $316 \mathrm{MWH}$ and $656 \mathrm{HIV}-$ Multicenter AIDS Cohort Study participants $\geq 40$ years at baseline, with neuropsychological testing every 2 years and concurrent BMI and WC measurements, were included. MWH were included if taking $\geq 2$ antiretroviral agents and had HIV-1 RNA $<400$ copies $/ \mathrm{mL}$ at $>80 \%$ of visits. Mixed-effects models included all visits from 1996 to 2015, stratified by HIV serostatus, and adjusted for sociodemographic, behavioral, and clinical characteristics. At baseline and follow-up, $8 \%$ of $\mathrm{MWH}$ and $15 \%$ of HIV- men and $41 \%$ of $\mathrm{MWH}$ and $56 \%$ of HIV- men were $\geq 60$ years, respectively.

\section{Results}

Cross-sectionally, higher BMI was inversely associated with motor function in MWH and HIVmen, and attention/working memory in HIV- men. WC was inversely associated with motor function in MWH and HIV- men. Longitudinal associations indicated an obese BMI was associated with a less steep decline in motor function in MWH whereas in HIV- men, obesity was associated with a greater decline in motor function, learning, and memory. WC, or central obesity, showed similar patterns of associations.

\section{Conclusion}

Higher adiposity is associated with lower cognition cross-sectionally and greater cognitive decline, particularly in HIV- men. Overweight and obesity may be important predictors of neurologic outcomes and avenues for prevention and intervention.

\author{
Correspondence \\ Dr. Rubin \\ Irubin1@jhmi.edu
}

\section{RELATED ARTICLE}

\section{Editorial}

Could excess body weight be good for cognitive health in chronic HIV infection?

Page 95 


\section{Glossary}

$\mathbf{A D}=$ Alzheimer disease $\mathbf{A R V}=$ antiretroviral; $\mathbf{B M I}=$ body mass index; CalCAP $=$ California Computerized Assessment Package; GPEG = Grooved Pegboard; HIV- = HIV-uninfected; MACS = Multicenter AIDS Cohort Study; MCI = mild cognitive impairment; $\mathbf{M W H}=$ men with HIV; PWH = people with HIV; RAVLT = Rey Auditory Verbal Learning Test; SDMT $=$ Symbol Digit Modalities Test $;$ TMT $=$ Trail-Making Test $; \mathbf{W C}=$ waist circumference.

\section{Is Obesity Associated with Cognitive Decline in Men with HIV?}

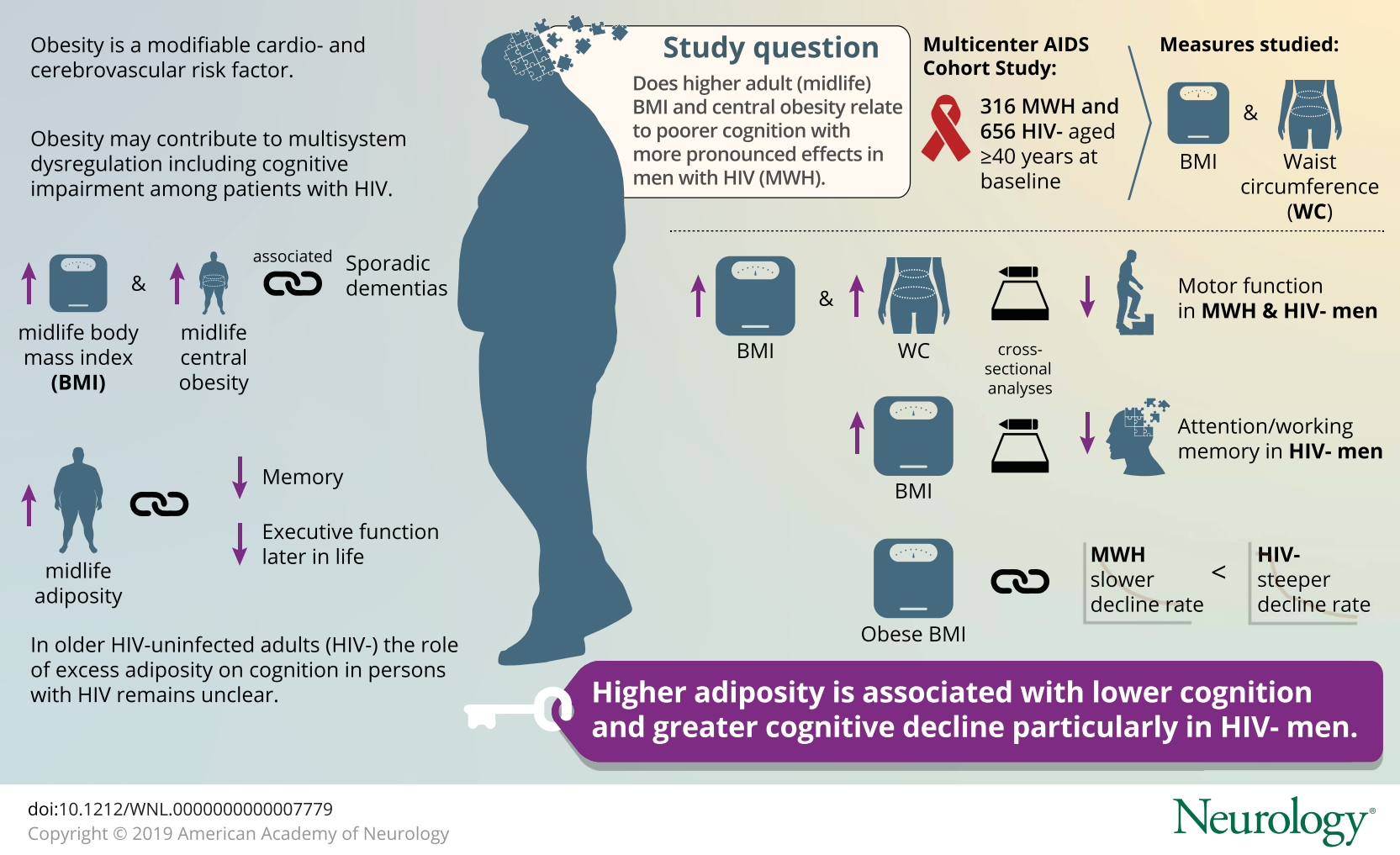

The potency and effectiveness of contemporary antiretrovirals (ARVs) has yielded an older and aging HIV population. Concomitantly, the prevalence of age- and aging-related diseases (e.g., cardiovascular and cerebrovascular comorbidities) is increasing and these factors contribute to adverse neurologic consequences such as cognitive impairment. Modifiable vascular risk factors, such as obesity, are common among people with HIV (PWH $)^{1,2}$ in proportions similar to those observed via national US estimates. ${ }^{3}$ The high prevalence ${ }^{1,2}$ or underlying causes of excess adiposity (e.g., ARVs, ${ }^{4}$ food insecurity ${ }^{5}$ ) in PWH may be strong contributors to cognitive impairment. Among HIV-uninfected (HIV-) adults, higher midlife body mass index (BMI) and central obesity (e.g., waist circumference [WC]) are associated with a higher risk of late-life, sporadic dementias, including Alzheimer disease, which are typically diagnosed at age 65 years or older. ${ }^{6-11}$ In addition, longer exposure to adiposity in midlife is associated with decreased memory at 60-64 years of age, ${ }^{12}$ and higher midlife adiposity is associated with lower executive function 10 years later. ${ }^{13}$

In $\mathrm{PWH}$, less is known about the role of excess adiposity on cognition. Cross-sectional analyses focusing on younger adults indicates obesity is associated with global cognitive impairment ${ }^{14-16}$ and slower processing speed. ${ }^{17}$ Whether midlife obesity predicts cognitive impairment or decline similar to or greater than that in HIV-individuals is unknown. We examined the prospective association of adult BMI and WC on 10-year domain-specific cognitive change in men with HIV $(\mathrm{MWH})$ vs HIV- men. We hypothesized that higher adult BMI and WC would relate to poorer cognition with more pronounced effects among $\mathrm{MWH}$.

\section{Methods}

\section{Participants and data source}

The study population comprised MWH and HIV- men enrolled in the Multicenter AIDS Cohort Study (MACS), a prospective study of men with or at risk of HIV infection within the United States. The cohort includes participants at study sites located in Los Angeles; Pittsburgh/Columbus, Ohio; Baltimore/Washington, DC; and Chicago. The institutional review boards at each site approved study protocols and forms, and each participant provided written informed consent. 


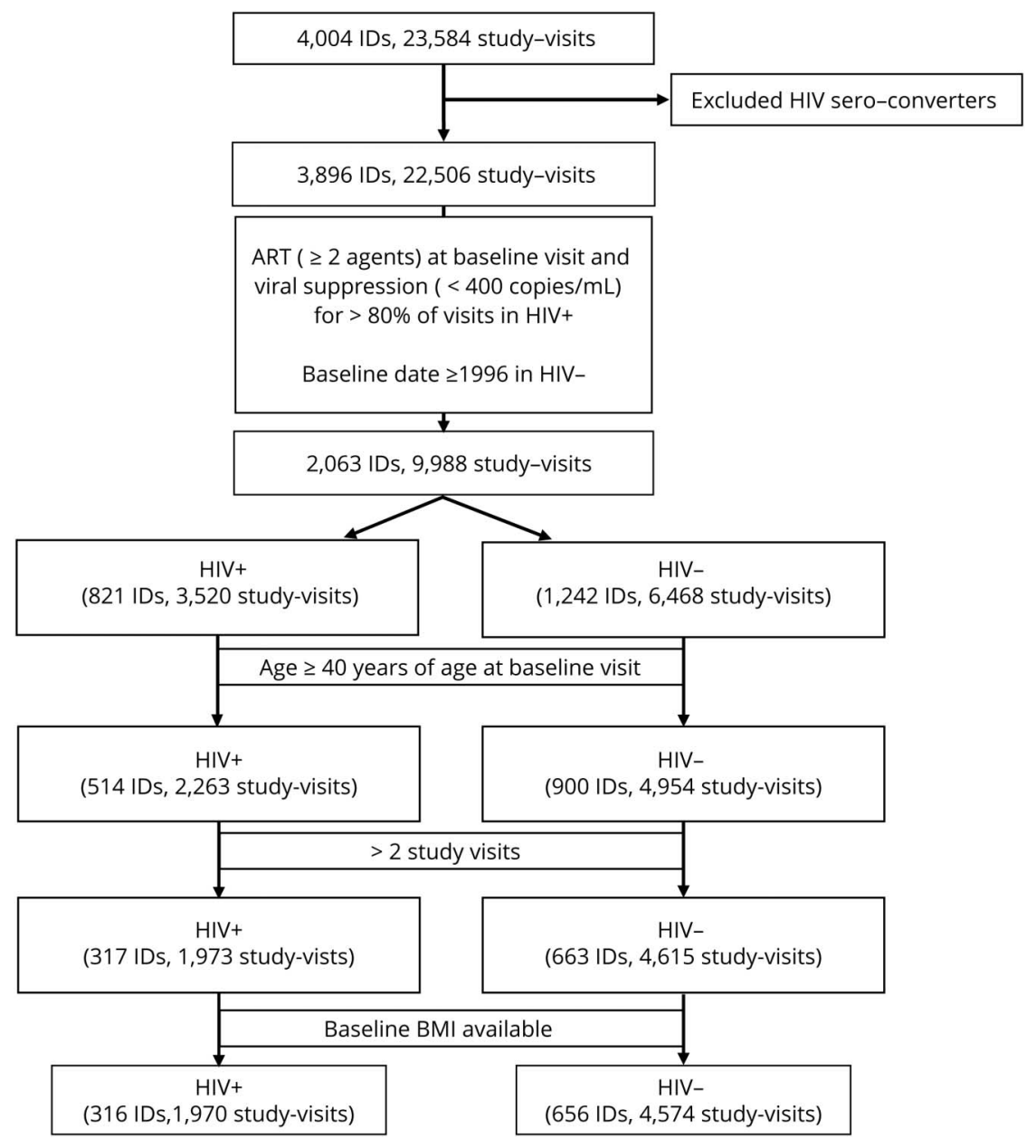

$\mathrm{ART}=$ antiretroviral therapy; $\mathrm{BMI}=$ body mass index.

For these analyses, participant eligibility was based on (1) age $\geq 40$ years; (2) documented completion of the full neuropsychological test battery (thus no missing data) at a minimum of 2 visits from 1996 or later (considered baseline); and (3) no prior self-reported psychotic disorders or documented dementia. MWH also had to be on $\geq 2 \mathrm{ARV}$ agents with an HIV-1 RNA $<400$ copies/mL at $>80 \%$ of visits, a criterion chosen to represent those who were mostly well-controlled on therapy without excluding people that may have been off or failed therapy for short periods of time. Our goal was to leverage a contemporary clinically relevant population of treatment-adherent virally suppressed $\mathrm{MWH}$. Figure 1 shows how the analytic sample was achieved.

\section{Primary exposure variables}

BMI was calculated by dividing body weight (kilograms) by height (meters) squared and categorized as underweight $\left(<18.5 \mathrm{~kg} / \mathrm{m}^{2}\right)$, normal or healthy weight $\left(18.5-24.9 \mathrm{~kg} / \mathrm{m}^{2}\right)$, overweight $\left(25.0-29.9 \mathrm{~kg} / \mathrm{m}^{2}\right)$, and obese $\left(\geq 30 \mathrm{~kg} / \mathrm{m}^{2}\right)$. Underweight men $(\mathrm{n}=20)$ were excluded from analysis given their low representation $(<1 \%$ of men) and potential morbidities accompanying underweight. WC was measured in centimeters while in the standing position and categorized as central obesity when $\geq 102 \mathrm{~cm}$ (40 inches) vs $<102 \mathrm{~cm}$, a cutoff associated with high risk of cardiovascular disease or its risk factors (e.g., type 2 diabetes, hypertension). ${ }^{18} \mathrm{BMI}$ was available on all participants at the baseline visit; WC was available on 834 of the 972 participants (85\%).

\section{Neuropsychological outcomes}

A full neuropsychological test battery was administered to participants at baseline and every 2 years thereafter ${ }^{19}$ and included Rey Auditory Verbal Learning Test (RAVLT), Rey Complex Figure, Trail-Making Test (TMT) Parts A and B, Stroop Color-Interference Test, Symbol Digit Modalities Test (SDMT), California Computerized Assessment Package (CalCAP) Reaction Time Test, and Grooved Pegboard (GPEG). Demographically adjusted $\mathrm{T}$ scores for each outcome were combined into 6 cognitive domains ${ }^{20}$ : learning (RAVLT total learning, Rey immediate recall), memory (RAVLT delayed recall, Rey delayed recall), executive function (TMT-B, Stroop interference trial), processing speed (SDMT, Stroop color-naming trial), sustained attention and working memory (CalCAP mean simple and complex 
reaction time), and motor function (GPEG, dominant and nondominant hand).

\section{Covariates}

Enrollment wave, number of visits per participant during the follow-up period (to control for learning effect subsequent to baseline), and sociodemographic (age, race, years of formal education), behavioral, and clinical characteristics were considered as covariates. Behavioral factors included use of tobacco, alcohol, marijuana, stimulants (nonprescription, including cocaine and methamphetamine), and other illicit drugs (each dichotomized into use vs no use $\leq 6$ months previously). Clinical factors included hypertension (systolic blood pressure $>140 \mathrm{~mm} \mathrm{Hg}$, diastolic blood pressure $>90 \mathrm{~mm} \mathrm{Hg}$, or self-reported hypertension with use of medications); dyslipidemia (fasting total cholesterol $\geq 200 \mathrm{mg} / \mathrm{dL}$, low-density lipoprotein $\geq 130 \mathrm{mg} / \mathrm{dL}$, high-density lipoprotein $<40 \mathrm{mg} / \mathrm{dL}$, triglycerides $\geq 150 \mathrm{mg} / \mathrm{dL}$, or lipid-lowering medication use with self-reported clinical diagnosis); malignancy (any cancer diagnosis); presence of hepatitis $C$ virus RNA; and clinically relevant depressive symptoms (Center for Epidemiological Studies-Depression questionnaire; 16 cutoff). HIV-specific covariates included current CD4+ T-cell count, suppressed HIV-1 viral RNA (<400 copies/mL; the lower limit of assay detection varied across study interval), history of an AIDS-defining opportunistic illness or malignancy, use of ARVs with known adverse cognitive or body composition effects (efavirenz, stavudine), and ARV exposure duration. Covariates were selected for multivariable models based on significant univariate associations $(p<0.10)$ with at least one neuropsychological test battery outcome.

\section{Statistical analyses}

Linear and polynomial (quadratic) trend mixed effects models stratified by HIV status were conducted to assess the effect of adult BMI and WC on cognition at the initial assessment and over a median 10 years follow-up. Each model examined the interaction between BMI or WC and time, where time was operationally defined as the participant's age. We examined time both as a linear and quadratic trend; however, model fits were optimal when time was treated as linear. Adjustments were made for covariates associated with at least one neuropsychological test battery outcome at $p<0.10$. Time-invariant covariates included cohort entry, baseline age, race/ethnicity, education level, and number of visits per participant during the follow-up period. Time-varying covariates included use of tobacco, alcohol, marijuana, other drugs, and stimulants; depressive symptoms; and hypertension. Time-varying HIVrelated covariates included current CD4+ T-cell count, history of AIDS-defining illness, HIV RNA <400 copies/mL, and current use of efavirenz or stavudine. Interactions between baseline age and BMI or WC were examined to ensure that results were due to the influence of aging and not chronological age on cognitive trajectories. There were no significant interactions between baseline age and BMI or WC across models and therefore only baseline age was included in analyses. All models were conducted using SAS (version 9.4, Cary, NC).

\section{Data availability}

MACS de-identified data can be obtained by submitting a request to the Center for Analysis and Management of MACS for purposes of replicating procedures and results.

\section{Results}

A total of 972 (316 MWH and 656 HIV-) men aged 40-84 years at baseline were included. Of these, $8 \%$ of $\mathrm{MWH}$ and $15 \%$ of HIV - men were $\geq 60$ years old at baseline and $41 \%$ of MWH and $56 \%$ of HIV - men were $\geq 60$ years old at follow-up. On average, MWH were younger (mean 49.1 years, SD 6.6; range 40-72 years) than HIV- men (mean 51.4 years, SD 8.1; range 40-84 years; $p<0.001)$. MWH vs HIV- men were less likely to be white ( $63 \%$ vs $76 \% ; p<0.001$ ), have a graduate education ( $28 \%$ vs $38 \%$; $p<0.004)$, or have an obese BMI (12\% vs $20 \%$; $p<0.003)$.

Table 1 shows baseline characteristics as a function of BMI category and HIV status. Among MWH and HIV- men, hypertension was more common among those with an obese BMI followed by overweight and normal BMI ( $p s<0.05$ ). Among $\mathrm{MWH}$, tobacco use was less common among overweight vs those with a normal or obese BMI $(p=0.04)$. Use of efavirenz was more common among those with a normal BMI vs overweight or obese BMI $(p=0.04)$. Among HIV- men, black participants were more likely to be obese $\left(>30 \mathrm{~kg} / \mathrm{m}^{2}\right)$ vs having an overweight or normal BMI $(p=0.04)$. Marijuana use was less common among those with an obese BMI vs those with overweight or normal BMI $(p=0.04)$.

\section{Cross-sectional association of BMI and WC with cognition}

Among MWH, BMI was only associated with motor function (table 2). MWH with an obese BMI performed worse than MWH with an overweight BMI $(p=0.04)$. The same pattern was seen for central obesity $(p=0.03)$. Among HIV- men, higher BMI was inversely associated with attention/working memory and motor function (table 2). On attention/working memory, HIV- men with an obese BMI performed worse than those with an overweight or normal BMI $(p s<0.05)$. Regarding motor function, HIV- men with an obese and overweight BMI performed worse than those with a normal BMI $(p s<0.05)$. Central obesity was only associated with motor function $(p=0.02)$.

\section{Longitudinal association of BMI and WC with cognitive decline}

During the follow-up period, serial cognitive assessments for MWH were performed at a median (range) of 5 (3-26) visits over a median (range) of $9.8(1-24)$ years. There were no differences in number of follow-up visits $(p=0.53)$ or time from baseline $(p=0.67)$ by BMI status. The proportion of $M W H \geq 60$ at the final follow-up visit did not differ across BMI groups (range 39\%-43\%; $p=0.76$ ). Among HIV- men, serial cognitive assessments were performed at a median (range) of 
Table 1 Baseline characteristics of study population by HIV status (HIV-uninfected [HIV-], men with HIV [MWH]) and body mass index

\begin{tabular}{|c|c|c|c|c|c|c|c|c|}
\hline & \multicolumn{3}{|c|}{ HIV- $(n=656)$} & \multirow[b]{2}{*}{$p$ Value } & \multicolumn{3}{|l|}{ MWH $(n=316)$} & \multirow[b]{2}{*}{$p$ Value } \\
\hline & $\begin{array}{l}\text { Normal } \\
(n=245 \\
37 \%)\end{array}$ & $\begin{array}{l}\text { Overweight } \\
(n=281 \text {, } \\
43 \%)\end{array}$ & $\begin{array}{l}\begin{array}{l}\text { Obese } \\
(n=130\end{array} \\
20 \%)\end{array}$ & & $\begin{array}{l}\text { Normal } \\
(n=149,47 \%)\end{array}$ & $\begin{array}{l}\text { Overweight } \\
(n=129 \\
41 \%)\end{array}$ & $\begin{array}{l}\begin{array}{l}\text { Obese } \\
(n=38\end{array} \\
12 \%)\end{array}$ & \\
\hline Age, y, mean (SD) & $50.7(8.6)$ & $51.9(7.9)$ & $51.6(7.4)$ & 0.21 & $49.3(7.2)$ & $49.3(6.3)$ & $48.0(5.1)$ & 0.50 \\
\hline$\geq 60 y$ at baseline & $36(15)$ & $43(15)$ & $20(15)$ & 0.98 & $17(11)$ & $9(7)$ & $0(0)$ & 0.98 \\
\hline$\geq 60$ y at follow-up & $124(51)$ & $164(58)$ & $77(59)$ & 0.13 & $64(43)$ & $50(39)$ & $15(40)$ & 0.76 \\
\hline Calendar year of first visit (SD) & $2003(4.0)$ & $2004(3.6)$ & $2003(3.5)$ & 0.01 & $2004(4.5)$ & $2004(4.4)$ & $2006(5.2)$ & 0.15 \\
\hline Time from baseline, y (SD) & $10.6(4.8)$ & $10.2(4.3)$ & $10.1(4.1)$ & 0.48 & $9.9(4.9)$ & $9.9(4.5)$ & $9.2(5.6)$ & 0.67 \\
\hline No. of visits per participant (SD) & $7.5(4.9)$ & $6.5(3.6)$ & $6.9(4.0)$ & 0.04 & $6.2(3.7)$ & $6.4(3.6)$ & $5.7(3.0)$ & 0.53 \\
\hline Body mass index, $\mathrm{kg} / \mathrm{m}^{2}$ (SD) & $22.8(1.5)$ & $27.1(1.3)$ & $34.0(4.4)$ & $<0.001$ & $22.7(1.6)$ & $27.0(1.4)$ & $33.4(3.2)$ & $<0.001$ \\
\hline Waist circumference, cm (SD) & $86.3(6.7)$ & $97.4(7.4)$ & $114(13.2)$ & $<0.001$ & $85.4(7.0)$ & $95.0(6.6)$ & $112(10.4)$ & $<0.001$ \\
\hline Enrolled after 2001 & $70(29)$ & $54(19)$ & $42(32)$ & 0.006 & $70(47)$ & $70(54)$ & $23(60)$ & 0.24 \\
\hline \multicolumn{9}{|l|}{ Race } \\
\hline White & $184(75)$ & $224(79)$ & $89(68)$ & 0.04 & $100(67)$ & $78(60)$ & $20(53)$ & 0.21 \\
\hline Black & $40(16)$ & $44(16)$ & $33(25)$ & 0.04 & $39(26)$ & $39(30)$ & $15(39)$ & 0.27 \\
\hline Other & $21(8)$ & $13(5)$ & $8(6)$ & 0.18 & $10(7)$ & $12(9)$ & $3(8)$ & 0.73 \\
\hline \multicolumn{9}{|l|}{ Education } \\
\hline High school & $27(11)$ & $36(13)$ & $20(15)$ & 0.49 & $29(19)$ & $20(15)$ & $9(23)$ & 0.46 \\
\hline Any college & $121(49)$ & $143(51)$ & $60(46)$ & 0.67 & $73(49)$ & $71(55)$ & $24(63)$ & 0.25 \\
\hline Graduate & $96(39)$ & $102(36)$ & $50(38)$ & 0.76 & $47(31)$ & $38(29)$ & $5(13)$ & 0.08 \\
\hline \multicolumn{9}{|l|}{ Tobacco use } \\
\hline Never & $71(29)$ & $104(37)$ & $41(31)$ & 0.14 & $47(31)$ & $37(29)$ & $9(23)$ & 0.62 \\
\hline Former & $106(43)$ & $118(42)$ & $56(43)$ & 0.95 & $44(29)$ & $60(46)$ & $15(39)$ & 0.01 \\
\hline Current & $67(27)$ & $58(21)$ & $33(25)$ & 0.18 & $58(39)$ & $32(25)$ & $14(37)$ & 0.04 \\
\hline \multicolumn{9}{|l|}{ Alcohol use (drinks/week) } \\
\hline 0 & $33(13)$ & $44(16)$ & $25(19)$ & 0.35 & $31(20)$ & $34(26)$ & $9(24)$ & 0.55 \\
\hline $1-3$ & $106(43)$ & $131(47)$ & $62(48)$ & 0.66 & $85(57)$ & $63(49)$ & $18(47)$ & 0.31 \\
\hline $4-13$ & $81(33)$ & $81(29)$ & $33(25)$ & 0.26 & $22(15)$ & $21(16)$ & $9(24)$ & 0.42 \\
\hline$>13$ & $24(10)$ & $24(8)$ & $10(8)$ & 0.76 & $11(7)$ & $11(8)$ & $2(5)$ & 0.79 \\
\hline \multicolumn{9}{|l|}{ Drug use } \\
\hline Marijuana & $75(31)$ & $74(26)$ & $24(18)$ & 0.04 & $49(33)$ & $38(29)$ & $12(32)$ & 0.83 \\
\hline Other drug & $35(14)$ & $57(20)$ & $17(13)$ & 0.08 & $24(16)$ & $32(25)$ & $5(13)$ & 0.11 \\
\hline Stimulant use & $29(12)$ & $37(13)$ & $11(8)$ & 0.38 & $23(15)$ & $19(15)$ & $3(8)$ & 0.48 \\
\hline Hepatitis C virus & $13(5)$ & $15(5)$ & $7(5)$ & 0.99 & $20(13)$ & $11(8)$ & $3(8)$ & 0.35 \\
\hline Depression & $40(16)$ & $56(20)$ & $30(23)$ & 0.27 & $38(26)$ & $28(22)$ & $15(39)$ & 0.09 \\
\hline Hypertension & $53(22)$ & $113(40)$ & $76(58)$ & $<0.001$ & $47(31)$ & $51(39)$ & $21(55)$ & 0.02 \\
\hline \multicolumn{9}{|l|}{ HIV-specific factors } \\
\hline \multicolumn{9}{|l|}{ CD4+ T-cell count, cells/ $\mu \mathrm{L}$} \\
\hline$<200$ & - & - & - & - & $15(10)$ & $6(5)$ & 1 (3) & 0.12 \\
\hline
\end{tabular}


Table 1 Baseline characteristics of study population by HIV status (HIV-uninfected [HIV-], men with HIV [MWH]) and body mass index (continued)

\begin{tabular}{|c|c|c|c|c|c|c|c|c|}
\hline & \multicolumn{3}{|c|}{ HIV $-(n=656)$} & \multirow[b]{2}{*}{$p$ Value } & \multicolumn{3}{|l|}{ MWH $(n=316)$} & \multirow[b]{2}{*}{$p$ Value } \\
\hline & $\begin{array}{l}\text { Normal } \\
(n=245, \\
37 \%)\end{array}$ & $\begin{array}{l}\text { Overweight } \\
(n=281 \\
43 \%)\end{array}$ & $\begin{array}{l}\begin{array}{l}\text { Obese } \\
(n=130, \\
20 \%)\end{array}\end{array}$ & & $\begin{array}{l}\text { Normal } \\
(n=149,47 \%)\end{array}$ & $\begin{array}{l}\text { Overweight } \\
(n=129 \\
41 \%)\end{array}$ & $\begin{array}{l}\text { Obese } \\
(n=38 \\
12 \%)\end{array}$ & \\
\hline $200-499$ & - & - & - & - & $58(39)$ & $45(35)$ & $12(32)$ & 0.69 \\
\hline$>500$ & - & - & - & - & $76(51)$ & $77(60)$ & $24(65)$ & 0.17 \\
\hline Suppressed HIV-1 RNA & - & - & - & - & $140(94)$ & $119(92)$ & $33(86)$ & 0.34 \\
\hline History of AIDS & - & - & - & - & $21(14)$ & $8(6)$ & $2(5)$ & 0.05 \\
\hline Use of efavirenz & - & - & - & - & $47(31)$ & $24(19)$ & $9(24)$ & 0.04 \\
\hline Use of stavudine & - & - & - & - & $27(18)$ & $20(15)$ & $5(13)$ & 0.72 \\
\hline
\end{tabular}

Data presented as n (\%) except where indicated.

6 (3-26) visits over a median (range) of 10.4 (1-21) years. Among HIV- men, those with a normal or obese BMI had more follow-up visits compared to those with an overweight BMI ( $p s<0.05)$; however, time from baseline did not differ across BMI groups $(p=0.48)$. The proportion of HIV- men $\geq 60$ years of age at the final follow-up visit did not differ across BMI groups (range 51\%-59\%; $p=0.13$ ).
Among MWH, those with an obese BMI demonstrated less decline in motor function with increasing age vs those with a normal $(p=0.04)$ or overweight BMI $(p=0.05)$ (table 3; figure 2). A similar pattern was observed between central obesity and motor function $(p=0.07)$ (figure 3). Among HIV- men, those with an obese BMI demonstrated greater decline in memory, learning, and motor function with

Table 2 Differences in baseline cognitive function by baseline overweight or obesity measured using body mass index (BMI) and waist circumference (WC) among HIV-uninfected (HIV-) and men with HIV (MWH)

\begin{tabular}{|c|c|c|c|c|c|c|}
\hline \multirow[b]{2}{*}{ Primary predictors } & \multicolumn{6}{|c|}{ Cognitive domains, $\beta$ (SE) } \\
\hline & Memory & Learning & $\begin{array}{l}\text { Attention/working } \\
\text { memory }\end{array}$ & Executive function & Processing speed & Motor function \\
\hline \multicolumn{7}{|l|}{$\mathrm{BMI}^{\mathrm{a}}$} \\
\hline \multicolumn{7}{|l|}{ HIV- } \\
\hline Overweight (vs normal) & $-0.8(0.8)$ & $-0.9(0.7)$ & $-0.4(0.8)$ & $-0.2(0.8)$ & $0.0(0.8)$ & $-2.0(0.9)^{*}$ \\
\hline Obese (vs normal) & $0.0(0.9)$ & $0.0(0.9)$ & $-2.2(0.9)^{*}$ & $-1.4(0.9)$ & $-0.8(1.0)$ & $-2.4(1.1)^{\star}$ \\
\hline Obese (vs overweight) & $0.8(0.9)$ & $0.9(0.9)$ & $-1.9(0.9)^{*}$ & $-1.7(0.9) \dagger$ & $-0.9(1.0)$ & $-0.4(1.1)$ \\
\hline \multicolumn{7}{|l|}{ MWH } \\
\hline Overweight (vs normal) & $0.8(1.0)$ & $1.8(1.0) \ddagger$ & $0.1(1.1)$ & $-0.2(1.1)$ & $0.9(1.1)$ & $0.8(1.2)$ \\
\hline Obese (vs normal) & $0.8(1.5)$ & $0.9(1.5)$ & $-1.0(1.6)$ & $-2.7(1.6) \S$ & $-1.7(1.6)$ & $-2.9(1.8)$ \\
\hline Obese (vs overweight) & $-0.1(1.5)$ & $-0.9(1.6)$ & $-1.1(1.6)$ & $-2.5(1.7)$ & $-2.6(1.6)$ & $-3.8(1.8)^{*}$ \\
\hline \multicolumn{7}{|l|}{$W^{b}$} \\
\hline \multicolumn{7}{|l|}{ HIV- } \\
\hline Higher (vs lower) & $-0.0(0.8)$ & $0.3(0.8)$ & $-1.3(0.8)$ & $-1.3(0.8)$ & $-1.0(0.8)$ & $-2.44(1.0)^{*}$ \\
\hline \multicolumn{7}{|l|}{ MWH } \\
\hline Higher (vs lower) & $2.3(1.5)$ & $1.6(1.5)$ & $-0.2(1.5)$ & $-2.0(1.6)$ & $-1.6(1.6)$ & $-3.7(1.7)^{*}$ \\
\hline \multicolumn{7}{|c|}{$\begin{array}{l}\text { a Normal BMI, } 20.00-24.99 \mathrm{~kg} / \mathrm{m}^{2} \text {; overweight, } 25.00-29.99 \mathrm{~kg} / \mathrm{m}^{2} \text {; obese, } \geq 30.00 \mathrm{~kg} / \mathrm{m}^{2} \text {. } \\
\text { b Higher WC, } \geq 102 \mathrm{~cm} \text { ( } 40 \text { inches). All models adjusted for study enrollment year, baseline age, race, education, Center for Epidemiologic Studies Depression } \\
\text { Scale, number of visits per participant over the follow-up period, use of tobacco, alcohol, marijuana, stimulants, or other drugs, and hypertension. Models } \\
\text { among MWH also included baseline CD4 count, suppressed HIV-1 RNA, history of AIDS, use of efavirenz, and use of stavudine. } \\
{ }_{\star} \neq<0.05 ; \dagger p=0.06 ; \neq p=0.07 ; \xi p=0.09 \text {. }\end{array}$} \\
\hline
\end{tabular}


Table 3 Multivariable adjusted differences in longitudinal change in domain-specific cognitive performance by baseline body mass index (BMI) and waist circumference (WC) status among HIV-uninfected (HIV-) and men with HIV $(\mathrm{MWH})$

\begin{tabular}{|c|c|c|c|c|c|c|}
\hline & \multicolumn{6}{|c|}{ Cognitive domains, $\beta$ (SE) } \\
\hline & Memory & Learning & $\begin{array}{l}\text { Attention/working } \\
\text { memory }\end{array}$ & Executive function & Processing speed & Motor function \\
\hline \multicolumn{7}{|l|}{$\mathrm{BMI}^{\mathrm{a}}$} \\
\hline \multicolumn{7}{|l|}{ HIV- } \\
\hline Overweight (vs normal) & $-0.4(0.4)$ & $-0.5(0.4)$ & $-0.3(0.4)$ & $-0.0(0.4)$ & $-0.4(0.4)$ & $-0.5(0.6)$ \\
\hline Obese (vs normal) & $-1.5(0.5)^{\star}$ & $-1.4(0.5)^{*}$ & $-1.0(0.5) \dagger$ & $-0.1(0.5)$ & $-0.0(0.5)$ & $-3.3(0.8) \ddagger$ \\
\hline Obese (vs overweight) & $-1.1(0.6) \S$ & $-0.9(0.5)^{\star \star}$ & $-0.6(0.5)$ & $-0.1(0.5)$ & $0.3(0.5)$ & $-2.7(0.8) \ddagger$ \\
\hline \multicolumn{7}{|l|}{ MWH } \\
\hline Overweight (vs normal) & $0.5(0.6)$ & $-0.1(0.6)$ & $0.9(0.7)$ & $0.7(0.7)$ & $0.6(0.7)$ & $0.1(1.0)$ \\
\hline Obese (vs normal) & $0.7(1.0)$ & $0.3(1.1)$ & $1.8(1.1)$ & $1.1(1.2)$ & $1.4(1.1)$ & $3.3(1.6) \S$ \\
\hline Obese (vs overweight) & $0.2(1.0)$ & $0.5(1.1)$ & $0.9(1.1)$ & $0.3(1.2)$ & $0.8(1.1)$ & $3.2(1.7) \dagger$ \\
\hline \multicolumn{7}{|l|}{$w c^{b}$} \\
\hline \multicolumn{7}{|l|}{ HIV- } \\
\hline Higher (vs lower) & $-0.9(0.5)+\dagger$ & $-1.1(0.5) \S$ & $-0.6(0.5)$ & $-0.2(0.4)$ & $-0.6(0.5)$ & $-2.7(0.7) \ddagger$ \\
\hline \multicolumn{7}{|l|}{ MWH } \\
\hline Higher (vs lower) & $0.2(0.9)$ & $0.1(0.1)$ & $-0.4(1.1)$ & $-0.8(1.2)$ & $0.3(1.2)$ & $3.0(1.7) \ddagger \ddagger$ \\
\hline \multicolumn{7}{|c|}{$\begin{array}{l}\text { a Normal BMI, } 20.00-24.99 \mathrm{~kg} / \mathrm{m}^{2} \text {; overweight, } 25.00-29.99 \mathrm{~kg} / \mathrm{m}^{2} ; \text { obese, } \geq 30.00 \mathrm{~kg} / \mathrm{m}^{2} \text {. } \\
\text { b Higher WC, } \geq 102 \mathrm{~cm} \text { (40 inches). All models adjusted for study enrollment year, baseline age, race, education, Center for Epidemiologic Studies Depression } \\
\text { Scale, number of visits per participant over the follow-up period, use of tobacco, alcohol, marijuana, stimulants, or other drugs, and hypertension. Models } \\
\text { among HIV-infected individuals also included baseline CD count, suppressed HIV-1 RNA, history of AIDS, use of efavirenz, and use of stavudine. } \\
{ }^{\star} p<0.01 ; t p=0.05 ; \ddagger p<0.001 ; \S p<0.05 ;{ }^{*} p=0.06 ; \dagger \dagger p=0.08 ; \ddagger \neq p=0.07 \text {. }\end{array}$} \\
\hline
\end{tabular}

increasing age vs those with a normal BMI $(p s<0.01)$ and similar pattern towards decline in attention/working memory $(p=0.05)$ (table 3; figure 2). HIV- men with an obese BMI also demonstrated greater decline on memory and motor function with increasing age vs those with an overweight BMI $(p s<0.05)$ and a similar pattern for decline in learning $(p=$ $0.06)$. A similar pattern was observed with central obesity (figure 3) ( $p s<0.05)$; and a similar pattern for decline in the memory domain $(p=0.08)$. Given that age-related declines for HIV- extend beyond those of MWH, we re-ran the significant effects in HIV- men restricting the age to the same maximum as observed in MWH ( $\leq 70$ years of age). Similar patterns of association were observed.

\section{Discussion}

Higher adiposity was associated with lower baseline cognitive performance in MWH and HIV- men. Specifically, higher adiposity was associated with lower motor function in both groups and attention/working memory in HIV- men. Although baseline cross-sectional adiposity-cognition associations were similar between MWH and HIV- men, longitudinal associations differed by HIV status. Higher adiposity was associated with greater cognitive decline over the average 10-year follow-up period in HIV- men whereas higher adiposity tended to be protective against cognitive decline, notably motor function in MWH. Clinically, cross-sectional BMI group differences among $\mathrm{MWH}$ and HIV- men ranged from small to medium effect sizes (2- to 4-point $\mathrm{T}$ score differences or 0.2-0.4 SDs) whereas longitudinal associations were larger, particularly for motor function among HIV- men. By 60 years of age, obese HIV- men were $1 \mathrm{SD}$ below the mean T score $(\sim 40)$, and by 70 years of age, were 2 SDs below the mean $T$ score $(\sim 30)$, suggestive of possible adiposity-associated influences on brain aging.

Reasons for different patterns of baseline BMI and cognitive change over time by HIV status are multifold. Among MWH, numerous factors may influence the BMI-cognition link. First, $\mathrm{MWH}$ may have experienced an initiatory cognitive hit due to HIV/AIDS (legacy effect), thus leaving minimal influence of BMI in comparison. Second, MWH have experienced diverse histories of ARV use in terms of duration and type of regimen in response to changing HIV infection severity over time. Third, MWH may have additional comorbidities (e.g., mental health and substance use disorders) and adverse lifestyle characteristics and utilize numerous nonARVs (e.g., antidepressants) that influence the association between BMI and cognition. However, while multivariable- 
Figure 2 Multivariable adjusted longitudinal change in domain-specific cognitive performance as a function of baseline body mass index (BMI) among HIV-uninfected (HIV-) and men with HIV (MWH)

Memory

A. HIV-

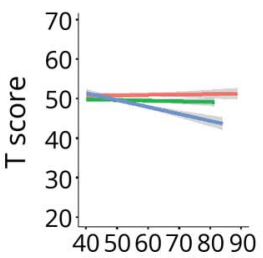

Learning

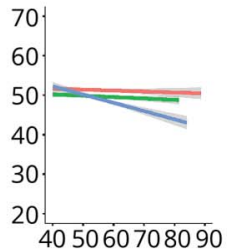

Attention/working memory

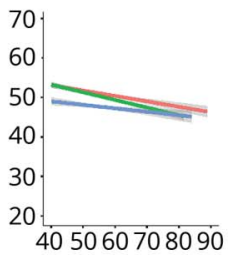

Executive function

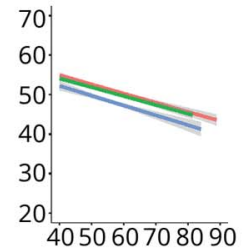

Baseline BMI status

- Normal

- Overweight

- Obese

Motor function
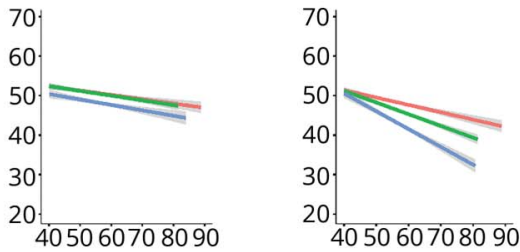

\section{B. $\mathrm{MWH}$}
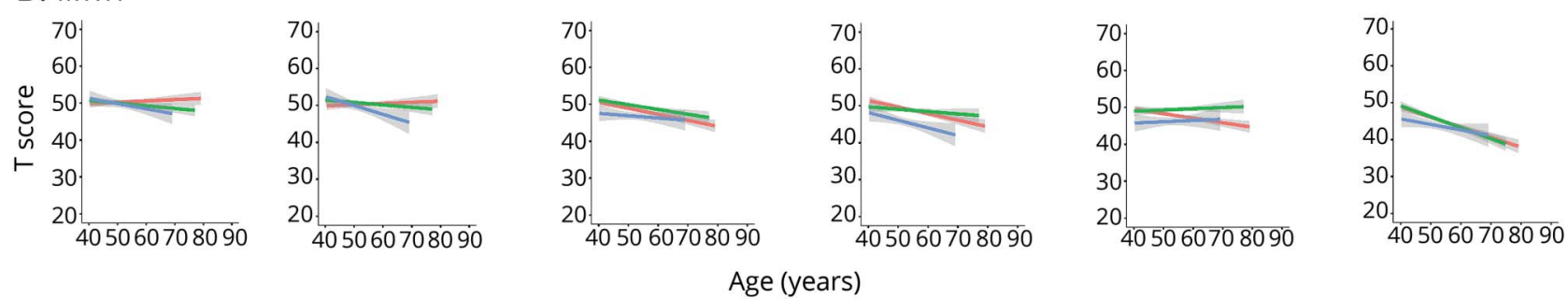

(A) HIV-. (B) MWH. All models adjusted for study enrollment year, baseline age, race, education, Center for Epidemiologic Studies Depression Scale, number of visits per participant, use of tobacco, alcohol, marijuana, stimulants, or other drugs, and hypertension. Models among HIV-infected individuals also included baseline CD4 count, suppressed HIV-1 RNA, history of AIDS, use of efavirenz, and use of stavudine.

adjusted analyses were used, together, these factors may contribute to dysregulation of metabolic, ${ }^{21}$ vascular, ${ }^{22}$ and cognitive health. ${ }^{23}$ Fourth, among HIV- men, obesity is associated with factors that might have additional effects on cognition and motor function (low motivation, poor dexterity) compared to MWH. Fifth, lack of associations within specific domains among $\mathrm{MWH}$ could be due to less statistical power, since twice as many HIV- men contributed data compared to MWH. Finally, the number of obese MWH is smaller, thus estimates are less precise than for HIV- men.

Evaluating the association between overweight and obesity and cognitive performance and decline in this sample is predicated on published literature showing an association of higher mid-late BMI or central obesity with late-onset, sporadic Alzheimer disease $(\mathrm{AD})$, vascular dementia, and total dementia risk in uninfected adults. ${ }^{24}$ An association between higher $\mathrm{BMI}$ and $\mathrm{AD}$ in 70-year-old women followed for at least 10 years was first reported in $2003 .^{6}$ More consistent associations between midlife, vs later-life, overweight and obesity followed. ${ }^{24}$ These results have not been replicated in aging adults with HIV infection. In addition, several epidemiologic studies suggest a paradoxical association of BMI with clinical dementia onset. ${ }^{25-27}$ Using traditional categories of BMI, studies report that an overweight or obese BMI or central adiposity in midlife may increase $\mathrm{AD}$ and dementia risk in later life. ${ }^{7-9,24,28-31}$ Subsequently, a decline in BMI over years in later life ${ }^{32}$ (e.g., $\geq 65$ years) may precede a dementia diagnosis, and may be characteristic of prodromal or preclinical dementia ${ }^{33-36}$ or accompany dementia onset. ${ }^{24,37,38}$ Evidence of BMI decline during prodromal dementias also comes from observations of unintentional body weight loss from mid- to late-life increasing risk for mild cognitive impairment $(\mathrm{MCI})^{39}$ and more rapid progression of cognitive decline among those with mild dementia. ${ }^{32}$ Elderly individuals with MCI also present with a lower BMI compared to their cognitively intact peers ${ }^{40}$ and patients with MCI who are losing body weight show more accelerated dementia progression. ${ }^{32,41}$ Thus, BMI is intricately linked with cognitive impairments and dementias before and after a dementia diagnosis, and may lead to poorer health outcomes in dementia.

The role of BMI in cognitive performance and decline during midlife among $\mathrm{MWH}$ and HIV- men is less well-described and understood than the association between BMI and onset of late-onset, sporadic dementia. Cross-sectional studies report both lower and higher midlife BMI among those with lower levels of cognition. ${ }^{42}$ Longitudinal studies of BMI in association with cognitive change are also mixed, with results varying for BMI vs WC and by age. ${ }^{43,44}$ Reasons for the mixed literature are that there is no strict definition of midlife; there are other contributors to midlife cognitive fluctuations 
Figure 3 Multivariable adjusted longitudinal change in domain-specific cognitive performance as a function of baseline waist circumference (WC) among HIV-uninfected (HIV-) and men with HIV (MWH)

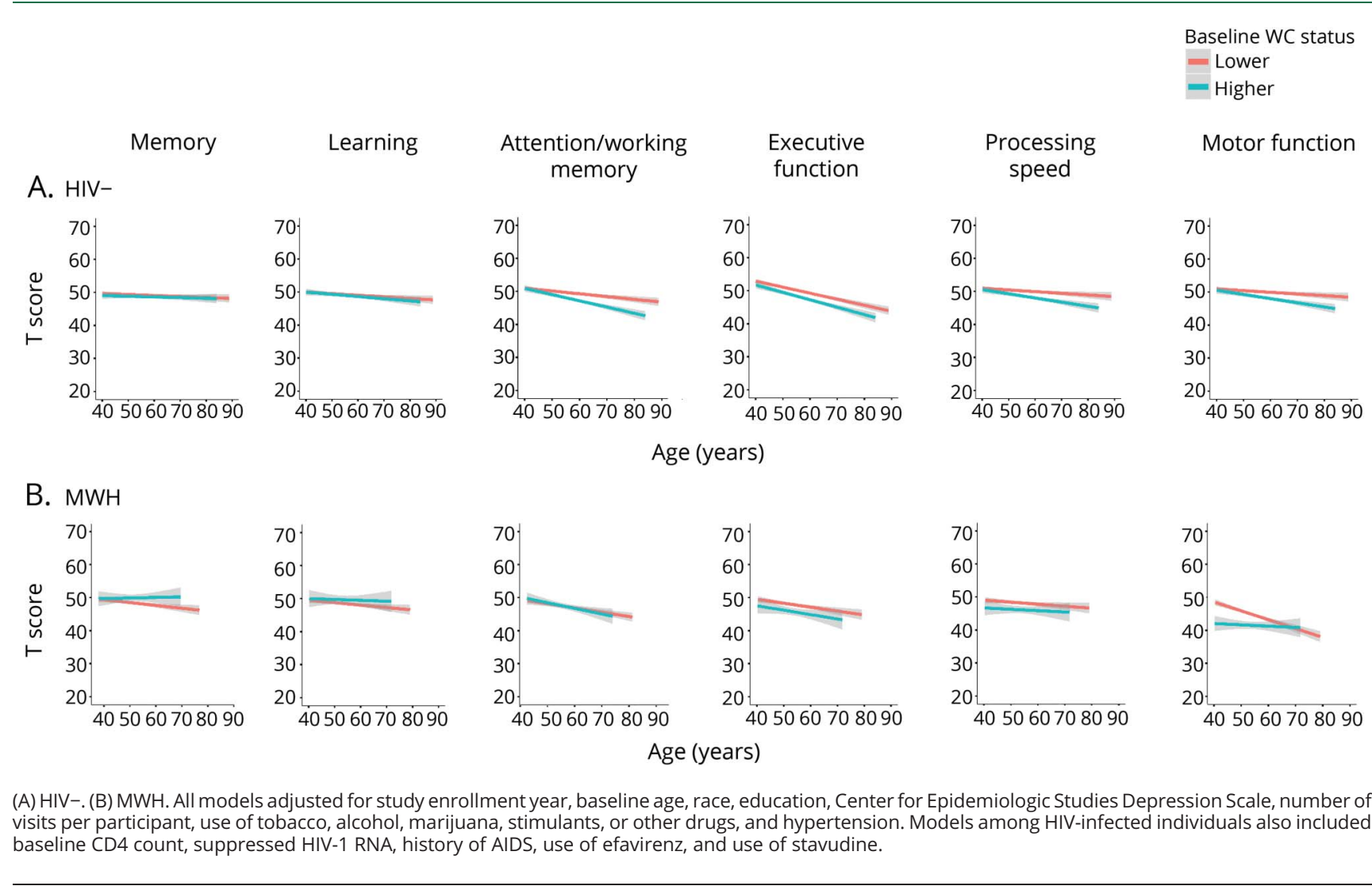

unrelated to future late-onset, sporadic dementia occurrence; risk factors for late-onset, sporadic cognitive impairments and dementias occur and accumulate over the life course; and populations at risk vary. ${ }^{45}$ Thus, the relative influences of vascular risk factors during the mid- to later-life transition, for example, ages 50-70 years, are difficult to evaluate in relation to cognition. This difficulty is accentuated in this understudied at-risk population, among whom thorough evaluation of vascular risk factors in association with cognition has not been reported.

There are several considerations when comparing cognitive data among younger adults without dementia, such as those presented here (only $13 \%$ of the sample $\geq 60$ at baseline), to those published in the literature of BMI and late-onset, sporadic dementias, the most severe form of cognitive impairment. First, this analysis includes an average of 10 years of continuous, frequent (every 6-24 months) follow-up. This is more frequent cognitive follow-up than seen in most aging and dementia studies. Second, the men in these analyses are, on average, at younger baseline and follow-up ages (early 50s-60s). Thus, the proportion of those with clinically relevant cognitive impairments is relatively low. Third, these men are transitioning through a life stage that has been described as dynamic for vascular risk factor trajectories. In HIVpopulation studies with lengthy follow-up from midlife to later life, one may expect to see an inflection point in the BMI trajectory such that, on average, adults increase in BMI until their 7 th or 8 th decade of life, followed by decline. ${ }^{46,47}$ However, this has not been consistently observed. ${ }^{36,48}$ Given the short duration of follow-up and relatively few men at this potential transition stage, whether an inflection point exists cannot be ascertained at this time. Fourth, we do not have MCI or dementia diagnoses in this analysis. While we are confident that there are no men with late-onset, sporadic dementia in the sample, there could be those with milder or $\mathrm{HIV}$-associated cognitive impairments. As the prevalence of HIV-associated neurocognitive disorder decreases in the era of effective ARVs ${ }^{49}$ and PWH reach older ages, their dementia risk may become similar to that observed in HIV- populations, albeit with excess comorbidities. ${ }^{50}$

Study limitations include use of formal years of education as a surrogate marker of premorbid general intelligence and cognitive reserve. Although a measure of premorbid general intelligence (Shipley Institute of Living Scale) was collected in the MACS, only $82 \%$ of the participants included in the analyses had these data available. To maximize our sample size, we decided to use years of formal education as a proxy. We were also unable to conduct additional analyses of obesity severity within the obesity category $\left(\geq 30 \mathrm{~kg} / \mathrm{m}^{2}\right)$. Of the 38 obese $\mathrm{MWH}$ and $130 \mathrm{HIV}$ - obese men, only 11 and 39, respectively, had BMI measures higher than Class I obesity $\left(30-34.9 \mathrm{~kg} / \mathrm{m}^{2}\right)$. Finally, while other statistical analysis 
frameworks are possible (e.g., structural equation modeling), we chose an approach that provided a means of comparing our findings to other published studies.

Our study demonstrates that obesity is associated with lower motor function in $\mathrm{MWH}$ and HIV- men, as well as greater cognitive decline among obese HIV- men compared to MWH. Domains of greatest decline included memory, learning, attention/working memory, and motor function. In addition, greater variability in cognitive trajectory was observed in association with adiposity among $\mathrm{MWH}$; however, obesity was associated with greater decline in motor function. Whether ARV use among MWH contributed to the attenuation of an association between obesity and cognitive decline in multiple cognitive domains cannot be elucidated.

Future priorities include the following. Longitudinal follow-up studies in the MACS are imperative to better describe life course trajectories of BMI, a modifiable vascular risk factor, in association with cognitive outcomes in both MWH and HIVmen. Replication studies are warranted to determine the generalizability of these findings to women with HIV and demographically similar HIV- women. Finally, these associations should be extended to measures of everyday functioning (e.g., medication and finance management) and quality of life to maximize ecological validity and clinical impact.

\section{Author contributions}

L.H.R., D.G., K.L.H., K.M.E.: conception and design of the study. L.H.R, L.Z.: acquisition and analysis of data. L.H.R, D.G., K.M.E.: drafting a significant portion of the manuscript or figures. All authors provided a critical review of the manuscript for important intellectual content and contributed to and approved the final manuscript.

\section{Acknowledgment}

Data used in this study were obtained from the Multicenter AIDS Cohort Study (MACS) database (aidscohortstudy.org/). The authors thank the MACS participants and investigators for their participation.

\section{Study funding}

Research reported in this publication was supported by the National Heart, Lung, and Blood Institute, the National Institute of Immunology, Allergy, and Infectious Disease, and the National Institute of Aging of the NIH under award numbers T32HL116276 to University of Colorado; K24 AI120834 to T.T.B.; NIA K23 AG050260 and R01AG054366 to K.M.E.; K23 AI110532 to J.E.L.; and P30MH075673 to L.H.R., N.S. Data in this manuscript were collected by the Multicenter AIDS Cohort Study (MACS). MACS (Principal Investigators): Johns Hopkins University Bloomberg School of Public Health (Joseph Margolick, Todd Brown), U01-AI35042; Northwestern University (Steven Wolinsky), U01-AI35039; University of California, Los Angeles (Roger Detels, Oto Martinez-Maza), U01-AI35040; University of Pittsburgh (Charles Rinaldo), U01-AI35041; the Center for Analysis and Management of
MACS, Johns Hopkins University Bloomberg School of Public Health (Lisa Jacobson, Gypsyamber D’Souza), UM1-AI35043. The MACS is funded primarily by the National Institute of Allergy and Infectious Diseases, with additional cofunding from the National Cancer Institute, the National Institute on Drug Abuse, and the National Institute of Mental Health. Targeted supplemental funding for specific projects was also provided by the National Heart, Lung, and Blood Institute and the National Institute on Deafness and Communication Disorders. MACS data collection is also supported by UL1-TR001079 (JHU ICTR) from the National Center for Advancing Translational Sciences (NCATS), a component of the NIH, and NIH Roadmap for Medical Research. The contents of this publication are solely the responsibility of the authors and do not represent the official views of the NIH, Johns Hopkins ICTR, or NCATS. The MACS website is located at aidscohortstudy.org/.

\section{Disclosure}

L.H. Rubin, D. Gustafson, K.L. Hawkins, L. Zhang, L.P. Jacobson, J.T. Becker, and C.A. Munro report no disclosures relevant to the manuscript. J.E. Lake has served as a consultant to Gilead Sciences and Merck and has received research funding from Gilead Sciences. E. Martin and A. Levine report no disclosures relevant to the manuscript. T.T. Brown served as a consultant to Gilead Sciences, Merck, BMS, ViiV Healthcare, Theratechnologies, and EMD-Serono. N. Sacktor reports no disclosures relevant to the manuscript. K.M. Erlandson has served as a consultant to Gilead Sciences and ViiV Healthcare and has received research funding from Gilead Sciences and Merck. Go to Neurology.org/N for full disclosures.

\section{Publication history}

Received by Neurology December 3, 2018. Accepted in final form March 11, 2019.

\section{References}

1. Crum-Cianflone N, Roediger MP, Eberly L, et al. Increasing rates of obesity among HIV-infected persons during the HIV epidemic. PLoS One 2010;5:e10106.

2. Amorosa V, Synnestvedt M, Gross R, et al. A tale of 2 epidemics: the intersection between obesity and HIV infection in Philadelphia. J Acquir Immune Defic Syndr 2005;39:557-561.

3. Hales CM, Carroll MD, Fryar CD, Ogden CL. Prevalence of Obesity Among Adults and Youth: United States, 2015-2016. Atlanta: NCHS, Center for Disease Control and Prevention; 2017.

4. Nduka CU, Uthman OA, Kimani PK, Stranges S. Body fat changes in people living with HIV on antiretroviral therapy. AIDS Rev 2016;18:198-211.

5. Sirotin N, Hoover DR, Shi Q Anastos K, Weiser SD. Food insecurity with hunger is associated with obesity among HIV-infected and at risk women in Bronx, NY. PLoS One 2014;9:e105957.

6. Gustafson D, Rothenberg E, Blennow K, Steen B, Skoog I. An 18-year follow-up of overweight and risk of Alzheimer disease. Arch Intern Med 2003;163: 1524-1528.

7. Anstey KJ, Cherbuin N, Budge M, Young J. Body mass index in midlife and late-life as a risk factor for dementia: a meta-analysis of prospective studies. Obes Rev 2011;12: e426-e37.

8. Whitmer RA, Gunderson EP, Quesenberry CP Jr, Zhou J, Yaffe K. Body mass index in midlife and risk of Alzheimer disease and vascular dementia. Curr Alzheimer Res 2007;4:103-109.

9. Whitmer RA, Gustafson DR, Barrett-Connor E, Haan MN, Gunderson EP, Yaffe K Central obesity and increased risk of dementia more than three decades later. Neurology 2008;71:1057-1064.

10. Gunstad J, Lhotsky A, Wendell CR, Ferrucci L, Zonderman AB. Longitudinal examination of obesity and cognitive function: results from the Baltimore Longitudinal Study of Aging. Neuroepidemiology 2010;34:222-229.

11. Tolppanen AM, Ngandu T, Kåreholt I, Laatikainen M, Soininen H, Kivipelto M. Midlife and late-life body mass index and late-life dementia: results from a prospective population-based cohort. J Alzheimers Dis 2014;38:201-209. 
12. Masi S, Georgiopoulos G, Khan T, et al. Patterns of adiposity, vascular phenotypes and cognitive function in the 1946 British Birth Cohort. BMC Med 2018;16:75.

13. Kesse-Guyot E, Andreeva VA, Touvier M, et al. Overall and abdominal adiposity in midlife and subsequent cognitive function. J Nutr Health Aging 2015;19:183-189.

14. Sattler FR, He J, Letendre S, et al. Abdominal obesity contributes to neurocognitive impairment in HIV-infected patients with increased inflammation and immune activation. J Acquir Immune Defic Syndr 2015;68:281-288.

15. McCutchan JA, Marquie-Beck JA, Fitzsimons CA, et al. Role of obesity, metabolic variables, and diabetes in HIV-associated neurocognitive disorder. Neurology 2012; 78:485-492.

16. Jumare J, El-Kamary SS, Magder L, et al. Body mass index and cognitive function among HIV-1 infected individuals in China, India and Nigeria. J Acquir Immune Defic Syndr 2019;80:e30-e35.

17. Okafor CN, Kelso NE, Bryant V, et al. Body mass index, inflammatory biomarkers and neurocognitive impairment in HIV-infected persons. Psychol Health Med 2017;22: 289-302.

18. Classification of overweight and obesity by BMI, waist circumference, and associated disease risks [online]. Available at: nhlbi.nih.gov/health/educational/lose_wt/BMI/ bmi_dis.htm. Accessed February 1, 2019.

19. Becker JT, Kingsley LA, Molsberry S, et al. Cohort profile: recruitment cohorts in the neuropsychological substudy of the Multicenter AIDS Cohort Study. Int J Epidemiol 2015;44:1506-1516.

20. Sacktor N, Skolasky RL, Seaberg E, et al. Prevalence of HIV-associated neurocognitive disorders in the Multicenter AIDS Cohort Study. Neurology 2016;86:334-340.

21. Azzoni L, Foulkes AS, Firnhaber C, et al. Metabolic and anthropometric parameter contribute to ART-mediated CD4+ T cell recovery in HIV-1-infected individuals: an observational study. J Int AIDS Soc 2011;14:37.

22. Becker JT, Kingsley L, Mullen J, et al. Vascular risk factors, HIV serostatus, and cognitive dysfunction in gay and bisexual men. Neurology 2009;73:1292-1299.

23. Vassallo M, Durant J, Biscay V, et al. Can high central nervous system penetrating antiretroviral regimens protect against the onset of HIV-associated neurocognitive disorders? AIDS 2014;28:493-501.

24. Emmerzaal TL, Kiliaan AJ, Gustafson DR. 2003-2013: a decade of body mass index, Alzheimer's disease, and dementia. J Alzheimers Dis 2015;43:739-755.

25. Arnoldussen IA, Kiliaan AJ, Gustafson DR. Obesity and dementia: adipokines interact with the brain. Eur Neuropsychopharmacol 2014;24:1982-1999.

26. Gustafson D, Bäckman K, Joas E, et al. A 37-year longitudinal follow-up of body mass index and dementia in women. J Alzheimers Dis 2012;28:162-171.

27. Kiliaan AJ, Arnoldussen IA, Gustafson DR. Adipokines: a link between obesity and dementia? Lancet Neurol 2014;13:913-923.

28. Kivipelto M, Ngandu T, Fratiglioni L, et al. Obesity and vascular risk factors at midlife and the risk of dementia and Alzheimer disease. Arch Neurol 2005;62:1556-1560.

29. Whitmer RA, Gunderson EP, Barrett-Connor E, Quesenberry CP Jr, Yaffe K. Obesity in middle age and future risk of dementia: a 27 year longitudinal population based study. BMJ 2005;330:1360-1364.

30. Beydoun MA, Beydoun HA, Wang Y. Obesity and central obesity as risk factors for incident dementia and its subtypes: a systematic review and meta-analysis. Obes Rev 2008;9:204-218.
31. Gustafson DR, Bäckman K, Waern M, et al. Adiposity indicators and dementia over 32 years in Sweden. Neurology 2009;73:1559-1566.

32. Besser LM, Gill DP, Monsell SE, et al. Body mass index, weight change, and clinical progression in mild cognitive impairment and Alzheimer disease. Alzheimer Dis Assoc Disord 2014;28:36-43.

33. Barrett-Connor E, Edelstein SL, Corey-Bloom J, Wiederholt WC. Weight loss precedes dementia in community-dwelling older adults. J Am Geriatr Soc 1996;44 $1147-1152$.

34. Buchman AS, Wilson RS, Bienias JL, Shah RC, Evans DA, Bennett DA. Change in body mass index and risk of incident Alzheimer disease. Neurology 2005;65:892-897.

35. Johnson DK, Wilkins $\mathrm{CH}$, Morris JC. Accelerated weight loss may precede diagnosis in Alzheimer disease. Arch Neurol 2006;63:1312-1317.

36. Knopman DS, Edland SD, Cha RH, Petersen RC, Rocca WA. Incident dementia in women is preceded by weight loss by at least a decade. Neurology 2007;69:739-746.

37. Atti AR, Palmer K, Volpato S, Winblad B, De Ronchi D, Fratiglioni L. Late-life body mass index and dementia incidence: nine-year follow-up data from the Kungsholmen project. J Am Geriatr Soc 2008;56:111-116.

38. Fitzpatrick AL, Kuller LH, Lopez OL, Diehr ES, Longstreth WT, Luchsinger JA. Midlife and late-life obesity and the risk of dementia: cardiovascular health study. Arch Neurol 2009;66:336-342.

39. Alhurani RE, Vassilaki M, Aakre JA, et al. Decline in weight and incident mild cog nitive impairment: Mayo Clinic study of aging. JAMA Neurol 2016;73:439-446.

40. Orsitto G. Different components of nutritional status in older inpatients with cognitive impairment. J Nutr Health Aging 2012;16:468-471.

41. Cova I, Clerici F, Rossi A, et al. Weight loss predicts progression of mild cognitive impairment to Alzheimer's disease. PLoS One 2016;11:e0151710.

42. Loprinzi PD, Frith E. Obesity and episodic memory function. J Physiol Sci 2018;68: 321-331.

43. Deckers K, Van Boxtel MPJ, Verhey FRJ, Kohler S. Obesity and cognitive decline in adults: effect of methodological choices and confounding by age in a longitudinal study. J Nutr Health Aging 2017;21:546-553.

44. Dye L, Boyle NB, Champ C, Lawton C. The relationship between obesity and cognitive health and decline. Proc Nutr Soc 2017;76:443-454.

45. Gustafson DR. Epidemiology informs clinical trials of cognitive impairments and lateonset dementias. J Neurol Neuromed Epub 2018 Sept 18.

46. Bäckman K, Joas E, Waern M, et al. 37 years of body mass index and dementia: effect modification by the APOE genotype: observations from the prospective population study of women in Gothenburg, Sweden. J Alzheimers Dis 2015;48:1119-1127.

47. Gustafson DR, Bäckman K, Joas E, Waern S, Guo X, Skoog I. 37 years of body mass index and dementia: observations from the prospective population study of women in Gothenburg, Sweden. J Alzheimers Dis 2012;28:163-171.

48. Stewart R, Masaki K, Xue QL, Peila H, White LR, Launer LJ. A 32-year prospective study of change in body weight and incident dementia: the Honolulu-Asia Aging Study. Arch Neurol 2005;62:55-60.

49. Sacktor N. Changing clinical phenotypes of HIV-associated neurocognitive disorders. J Neurovirol 2018;24:141-145.

50. Smail RC, Brew BJ. HIV-associated neurocognitive disorder. Handb Clin Neurol 2018;152:75-97. 